\title{
Phonon Scattering by Nanoscale Twin Boundaries
}

Jun Mao a, , Yumei Wang ${ }^{\mathrm{c}}$, Zihang Liu ${ }^{\mathrm{a}, \mathrm{d}}$, Binghui $\mathrm{Ge}^{\mathrm{c}}$, and Zhifeng Ren ${ }^{\mathrm{a}^{*}}$

${ }^{a}$ Department of Physics and Texas Center for Superconductivity, University of Houston, Houston, TX 77204, USA

${ }^{b}$ Department of Mechanical Engineering, University of Houston, Houston, TX 77204, $U S A$

${ }^{c}$ Beijing National Laboratory for Condensed Matter Physics, Institute of Physics, Chinese Academy of Sciences, P.O. Box 603, Beijing 100190, China

${ }^{d}$ National Key Laboratory for Precision Hot Processing of Metals and School of Materials Science and Engineering, Harbin Institute of Technology, Harbin 150001, China

*Corresponding author, email address: zren@uh.edu; 


\section{ABSTRACT}

Twins, as one kind of crystal defects, are often been observed in various material systems. However, its effect on phonon scattering is much less investigated comparing to other defects. The difficulty in understanding the phonon scattering effect by twins lies in the inevitable presence of other defects (e.g., grain boundaries), which overshadow the intrinsic scattering effect by twins. In order to resolve the issue, three different kinds of InSb specimens with microscale grains, nanoscale grains, and nanoscale grains with nanotwins were prepared in this work. By comparing the thermal conductivities between different specimens, our results unambiguously show that phonons can indeed be scattered by twin boundaries. However, in a striking contrast to grain boundaries, phonon scattering effect by twin boundaries is noticeably weaker, which could be ascribed to the coherent characteristics of twin boundaries.

Keywords: Phonon scattering; Nanotwins; Twin boundary; Thermal conductivity; $\mathrm{InSb}$ 


\section{INTRODUCTION}

Phonons can be effectively scattered by crystal defects and nanostructures, e.g., point defects [1-12], dislocations [13-18], grain boundaries [19-23], and nanoparticles/nanoprecipitates [24-32]. As one kind of defects, twins, which are often been observed in various material systems [33-38], its effect on phonon scattering is much less investigated comparing to other defects.

Theoretical investigations about phonon scattering by twins were reported recently, however the results were controversial [39-44]. Several reports predicted noticeable reduction of thermal conductivity $(\kappa)$ due to the presence of twins [39, 41-43]. Relationship between $\kappa$ and the spacing of twins, angle between the neighboring twins, and number of twins perpendicular to the direction of heat flow were reported $[39,41]$. In a striking contrast to the prediction of significant reduction in $\kappa$, a few reports pointed out that twin boundaries (TBs) can only lead to a weak phonon scattering effect $[40,44]$. In addition to the theoretical studies, there were only few experimental reports [45-47].

Therefore, the motivation of this work is to experimentally investigate the phonon scattering effect by twins. Due to the inevitable presence of other defects $(e . g$, grain boundaries, GBs), it is difficult to reveal the intrinsic phonon scattering effect by TBs. Besides, we are also interested in comparing the phonon scattering effect between the coherent TBs and non-coherent GBs. Therefore, three kinds of InSb specimens with microscale grains, nanoscale grains, and nanoscale grains with 
nanotwins were prepared in this work. Comparison of $\kappa$ between different specimens were made and phonon scattering effect by TBs and GBs were discussed.

\section{EXPERIMENTAL SECTION}

\section{Synthesis}

Specimens with microscale grains. Indium ingot (In, 99.98\%; Alfa Aesar), antimony pieces ( $\mathrm{Sb}, 99.8 \%$; Alfa Aesar) were weighed according to the stoichiometric composition. The raw materials with nominal composition were loaded into quartz tubes and were heated up to $923 \mathrm{~K}$ in 3 hours and kept for 3 hours and then quenched in water. The obtained ingots were hand-milled into powders by using agate mortar in glove box. Powders were then loaded into a graphite die with an inner diameter of $12.7 \mathrm{~mm}$ in glove box. Then, the graphite die with loaded powder was removed from glove box and immediately sintered by direct current-induced hot pressing at $768 \mathrm{~K}$ for $5 \mathrm{~min}$. The slightly higher hot pressing temperature for the specimen with microscale grains is necessary for densifying due to the much larger particle size. The hot pressed disks are about 2-3 mm thick.

Specimens with nanoscale grains. Identical melting method was used and the melted InSb ingot was ball milled for 10 hours at $1200 \mathrm{rpm}$. Powders were then hot pressed by the same procedure at $733 \mathrm{~K}$ for $5 \mathrm{~min}$. 
Specimens with nanoscale grains and nanotwins. The In and $\mathrm{Sb}$ elements were loaded into a stainless steel ball milling jar in glove box. After that, identical ball milling and hot pressing method was used to synthesize the specimens.

\section{Thermoelectric characterizations}

Specimens were cut into about $2 \mathrm{~mm} \times 2 \mathrm{~mm} \times 12 \mathrm{~mm}$ for simultaneous electrical resistivity and Seebeck coefficient characterizations under Helium atmosphere (ZEM-3; ULVAC Riko). Thermal conductivity $\kappa=d D C_{p}$ was calculated using the measured density $(d)$ by Archimedean method, specific heat $\left(C_{p}\right)$ by differential scanning calorimetry (DSC 404 C; Netzsch) and thermal diffusivity $(D)$ by laser flash method (LFA 457; Netzsch).

\section{Composition and nanostructure characterization}

Phase composition of the samples was characterized by X-ray diffraction (PANalytical X'pert PRO diffractometer with a $\mathrm{Cu} \mathrm{Ka}$ radiation source). Microstructures of the specimens were observed by a scanning electron microscope (SEM, JEOL 6330F) and a high resolution transmission electron microscope (HRTEM, JEOL 2100F). Specimens for electron microscope investigation were prepared by a standard procedure, including grinding, mechanical polishing, dimpling and then by ion-beam thinning at $3 \mathrm{kV}$ with liquid nitrogen cooling. HAADF-STEM 
experimental investigation was carried out using a JEM-ARM 200F TEM operated at $200 \mathrm{kV}$. The microscope was equipped with a Cold FEG source and double-sextupole Cs correctors for STEM and for TEM (for probe and for imaging), respectively. The attainable spatial resolution of the microscope is $80 \mathrm{pm}$. HAADF images were acquired at acceptance angles of 70-150 mrad. All of the images presented in this paper are Fourier-filtered to minimize the effect of the random noise.

\section{RESULTS AND DISCUSSION}

The obtained XRD results indicate that all the prepared InSb specimens are single phase without observable impurities within the detection limit of the machine (Fig. S1, supporting information). Fig. 1 shows the comparison of microstructures between InSb specimens prepared by different methods. The specimen with microscale grains (Fig. 1a) was prepared by melting and then hot pressing, the one with nanoscale grains (Fig. 1b) was prepared by ball milling the melted ingot and then hot pressing, and the one with nanoscale grains and nanotwins (GBs and TBs are marked by dotted lines and white arrows, respectively) was prepared by directly ball milling the elements and then hot pressing (Fig. 1c). Schematic views of the microstructures of different InSb specimens are also presented (Fig. 1d, 1e, and 1f).

The effect of ball milling process on the reduction of grain sizes is well-understood [48-50]. Formation of nanoscale twins by high energy ball milling is also not uncommon, which has been observed in ball-milled $\mathrm{Cu}$ [51], $\mathrm{Cu}-\mathrm{Ni}$ alloy [52] 
and $\mathrm{MgAgSb}$ [53]. The origin of twinning could be ascribed to the shear stress induced by ball milling that exceeds the critical shear stress for twinning; the grain size decreases to critical value below which twinning rather than slip is the preferred mode of deformation as well as the high strain rate of ball milling [51]. It is noted that although identical ball milling process was used, whether the twinning occurs or not is closely related to the choice of starting materials (In and Sb elements or melting InSb ingot). In our work, twinning occurs only when elements were used as starting material, and the reason for that is unclear at the current stage.

\section{Figure 1 should be here}

In order to clearly reveal the atomic-scale microstructural features of the twins (Fig. 1c), high resolution transmission electron microscope (HRTEM) and $C_{\mathrm{s}}$-corrected high angle annular dark field scanning transmission electron microscope (HAADF-STEM) investigations were carried out. Fig. 2a shows several nanotwins with spacing around $50 \mathrm{~nm}$ in one of the grains, where TBs are marked by white arrows. The rectangular region in Fig. $2 \mathrm{a}$ is enlarged in Fig. 2b, where two sharp TBs can be clearly observed. The magnification of one of the TBs in the inset of Fig. 2b clearly shows the crystal lattice and the orientation of neighboring twins. Fig. 2c and 2d show typical HRTEM images for nanotwins with different spacings. Fig. 2c show several nanotwins with spacing of $\sim 3 \mathrm{~nm}$, and Fig. $2 \mathrm{~d}$ shows multiple nanotwins with an even smaller spacing of $\sim 1.5 \mathrm{~nm}$. The observed nanoscale twins show a wide distribution of twin spacing in the specimen. There is a clear TB in Fig. 2e as pointed 
by the white arrows. The Fourier transform taken across the boundary between two domains are shown in the inset of Fig. 2e, which can be indexed as [1 10$]$ according to the $\bar{F} \overline{4} m$ cubic structure of InSb. It is the superposition of two sets of reflection spots from neighboring domains (marked separately by white and yellow circles). It can be concluded that the boundary is situated in (111) plane. The magnification of the rectangular region in Fig. 2e is shown in Fig. 2f, where atomically sharp and coherent TB can be clearly seen.

\section{Figure 2 should be here}

To investigate the phonon scattering effect by nanotwins, thermal conductivities of different specimens were measured. It should be pointed out that each kind of specimens were prepared by three batches and the measured $\kappa$ for different specimens showed good repeatability (Fig. S2, supporting information). Generally, $\kappa$ contains the electronic part $\left(\kappa_{\text {ele }}\right)$ and lattice part $\left(\kappa_{\text {lat }}\right)$. Among which, $\kappa_{\text {ele }}$ relates to the electrical resistivity $(\rho)$ according to the Wiedemann-Franz relation, $\kappa_{\text {ele }}=L T / \rho$, where $L$ is the Lorenz number and $T$ is the absolute temperature. Due to non-parabolic band of $\mathrm{InSb}$ [54], single Kane band model [55] is used for the calculation of $L$ (details of calculation can be found in supporting information). By subtraction of $\kappa_{\text {ele }}$ from the measured $\kappa, \kappa_{\text {lat }}$ can be obtained accordingly. Since $\kappa_{\text {lat }}$ is sensitive to the crystal defects, it could reveal the intrinsic phonon scattering effect by twin boundaries and grain boundaries.

Fig. 3 shows the comparison of $\kappa_{\text {lat }}$ between different InSb specimens and 
appreciable difference in $\kappa_{\text {lat }}$ is noted. The specimen with microscale grains shows substantially higher $\kappa_{\text {lat }}$ and the room temperature $\kappa_{\text {lat }}$ is $\sim 13.6 \mathrm{~W} \mathrm{~m}^{-1} \mathrm{~K}^{-1}$. Due to the large grain size, GBs are not effective in scattering the phonons. Therefore, only Umklapp scattering is the dominant scattering mechanism in this specimen. On the contrary, the specimen with nanoscale grains show a much lower $\kappa_{\text {lat }}$, the room temperature $\kappa_{\text {lat }}$ is only $\sim 8.2 \mathrm{~W} \mathrm{~m}^{-1} \mathrm{~K}^{-1}$, a reduction of $\sim 40 \%$. It clearly demonstrates that the nanoscale GBs can significantly scatter the phonons, and similar results have also been reported in various material systems $[20,21,23,56]$. Therefore, for the specimen with nanoscale grains, Umklapp scattering and grain boundary are both important. Calculated lattice thermal conductivity by considering the Umklapp scattering and GB scattering shows a reasonable agreement with the experimental results (Fig. S3, supporting information).

Comparing to the specimens with nanoscale grains, the specimens with both nanoscale grains and nanotwins show slightly lower $\kappa_{\text {lat }}$, the room temperature $\kappa_{\text {lat }}$ is $\sim 6.8 \mathrm{~W} \mathrm{~m}^{-1} \mathrm{~K}^{-1}$, a reduction of $\sim 17 \%$. This indicates that nanotwins could also scatter the phonons in InSb. Therefore, for the specimen with nanoscale grains and nanotwins, the dominant scattering mechanisms are Umklapp scattering, GB scattering, and TB scattering. Due to the simultaneous presence of nanoscale GBs and TBs in the prepared InSb specimens, it offers a good opportunity to compare the phonon scattering effect between the two different interfaces. By making the comparison of $\kappa_{\text {lat }}$ between the specimens, it is obvious that the phonon scattering effect by TBs is 
not as significant as the GBs. Our results are in a reasonable agreement with the theoretical calculations $[40,44]$, which indicate that TBs only lead to a weak phonon scattering effect.

\section{Figure 3 should be here}

To understand the distinct phonon scattering effect by GBs and TBs, detailed characterization of atomic structure around GBs is further conducted. As shown in Fig. 4a, a GB and several TBs are observed and the neighboring grains are marked by different colors. Magnified view of rectangular region 1 in Fig. 4a is shown in Fig. 4b, where multiple dislocations (marked by yellow circles) around the GB are observed. In order to distinctly show the structure of the dislocation core, filtering operation of Fig. $4 \mathrm{~b}$ was performed. From the filtered image shown in Fig. 4c, the inserted half planes are pointed out by yellow arrows. The region 3 in Fig. $4 \mathrm{~b}$ including two typical dislocations is magnified and rotated and shown in Fig. 4d. The Burgers vector was determined by making the closed clockwise Burgers circuit. The Burgers vector of one of the dislocations is determined to be $b=\frac{1}{3}[\overline{1} \overline{1} \overline{1}]$, which is the Frank partial dislocation. The other is Shockley partial dislocation with Burgers vector $b=\frac{1}{6}[\overline{1} \overline{12}]$. Fig. $4 \mathrm{e}$ shows the rotated and magnified micrograph of rectangular region 4 in Fig. 4b, where two intrinsic stacking faults (SFs) are marked by yellow dash lines. The capitals A, B, and $\mathrm{C}$ and lower-case $\mathrm{a}, \mathrm{b}$, and $\mathrm{c}$ denote the stacking sequence of $\mathrm{Sb}$ and In atomic layers. The stacking sequence of the perfect crystal 
structure of InSb is $\mathrm{aB} \rightarrow \mathrm{bC} \rightarrow \mathrm{cA}$ (the order is from bottom to top shown in Fig. 4e), while the stacking sequences with intrinsic SFs are $\mathrm{aB} \rightarrow \mathrm{bA} \rightarrow \mathrm{aB}$ (the ideal sequence should be $\mathrm{aB} \rightarrow \mathrm{bC} \rightarrow \mathrm{cA} \rightarrow \mathrm{aB}$, which means $\mathrm{C}$ and $\mathrm{c}$ layers have been extracted) and $\mathrm{bC} \rightarrow \mathrm{cB} \rightarrow \mathrm{bC}$ (the ideal sequence should be $\mathrm{bC} \rightarrow \mathrm{cA} \rightarrow \mathrm{aB} \rightarrow \mathrm{bC}$, which means $\mathrm{A}$ and a layers have been extracted). Fig. $4 \mathrm{f}$ shows the magnified view of rectangular region 2 in Fig. 4a, and the region close to GB is marked between two white dash lines. It is clear that the GB is not sharp and the region close to the GB extends over the range of several atoms. It is also noted that the triple junction is highly disordered. In a striking contrast to the GB, the observed TB is sharp and quite coherent. Therefore, due to the existence of crystal defects (e.g., dislocations, SFs) around the GB, and the disordered characteristic of the GB itself, the stress and strain around GB will be noticeably larger than those of the $\mathrm{TB}$, hence lead to much more significant phonon scattering effect. In other word, due to the coherent characteristic of the $\mathrm{TB}$, it shows appreciably weaker phonon scattering effect.

\section{Figure 4 should be here}

In addition to the difference in coherency, it can also be argued that since the thermal transmission should mainly be affected when the heat flux is perpendicular to the interfaces, while TBs are much more anisotropic than GBs, hence it is reasonable that weaker phonon scattering effect should be expected by TBs [44]. Besides, theoretical calculation shows that the twin boundaries can only slightly reduce the average phonon group velocity, therefore it will only lead to weak phonon scattering 
effect [44]. Furthermore, the coherent phonon heat conduction in superlattices has been reported recently, where low-frequency phonons could propagate through the entire superlattices and thus conduct heat coherently [57, 58]. It is worth noting that the structure of nanotwins is similar to that of the superlattice, therefore similar coherent phonon conduction at the twin boundaries can also be expected. In other words, twin boundary should demonstrate weak phonon scattering effect.

Besides, although we mainly focused on the phonon scattering effect by twin boundaries, it is noted that the measured electrical resistivity and Hall mobility (Fig. S4 and Tab. S1, supporting information) indicate that twin boundaries can also scatter the electrons in InSb specimens. The specimen with nanoscale grain and nanotwins shows slightly lower Hall mobility $\left(\mu \sim 117 \mathrm{~cm}^{2} \mathrm{~V}^{-1} \mathrm{~S}^{-1}\right)$ than that of one with nanoscale grains $\left(\mu \sim 135 \mathrm{~cm}^{2} \mathrm{~V}^{-1} \mathrm{~S}^{-1}\right)$.

\section{CONCLUSIONS}

In this work, three different kinds of InSb specimens with microscale grains, nanoscale grains, and nanoscale grains in conjunction with nanotwins were prepared. By comparing $\kappa_{\text {lat }}$ between different specimens, it is clear that nanotwins can indeed scatter the phonons and hence reduce the $\kappa_{\text {lat }}$. However, comparing to the nanoscale GBs, the phonon scattering by TBs is much weaker. It could be explained by the much smaller disorders in the TBs than that of the GBs. 


\section{ACKNOWLEDGEMENT}

Authors want to thank Dr. Cai Wen for his help for the stacking faults analysis. The work performed at the University of Houston is supported by the Solid State Solar-Thermal Energy Conversion Center ( $\left.\mathrm{S}^{3} \mathrm{TEC}\right)$, an Energy Frontier Research Center funded by the U.S. Department of Energy, Office of Science, Office of Basic Energy Sciences under Award Number: DE-SC0001299. Y. M. W. gratefully acknowledges the financial support from the National Natural Science Foundation of China (No. 11474329). 


\section{REFERENCES}

[1] M.C. Steele, F.D. Rosi, J. Appl. Phys. 29 (1958) 1517-1520.

[2] F.D. Rosi, B. Abeles, R.V. Jensen, J. Phys. Chem. Solids 10 (1959) 191-200.

[3] A.V. Ioffe, A.F. Ioffe, Sov. Phys. Solid State 2 (1960) 719-728.

[4] P.G. Klemens, Phys. Rev. 119 (1960) 507-509.

[5] P.G. Klemens, G.K. White, R.J, Tainsh. Philos. Mag. 7 (1962) 1323-1335.

[6] B. Abeles. Phys. Rev. 131 (1963) 1906-1911.

[7] Q. Shen, L. Chen, T. Goto, T. Hirai, J. Yang, G.P. Meisner, C. Uher, Appl. Phys. Lett. 79 (2001) 4165-4167.

[8] J. Yang, G.P. Meisner, L. Chen, Appl. Phys. Lett. 85 (2004) 1140-1142.

[9] Y. Pei, D.T. Morelli, Appl. Phys. Lett. 94 (2009) 122112.

[10]L. Hu, T. Zhu, X. Liu, X. Zhao, Adv. Funct. Mater. 24 (2014) 5211-5218.

[11]C. Fu, S. Bai, Y. Liu, Y. Tang, L. Chen, X. Zhao, T. Zhu, Nature Comm. 6 (2015) 8144.

[12]J. Mao, Y. Wang, B. Ge, Q. Jie, Z. Liu, U. Saparamadu, W. Liu, Z. Ren, Phys. Chem. Chem. Phys. 18 (2016) 20726-20737.

[13]R. Sproull, M. Moss, H. Weinstock, J. Appl. Phys. 30 (1959) 334-337.

[14]D. Kotchetkov, J. Zou, A. Balandin, D. Florescu, F.H. Pollak, Appl. Phys. Lett. 79 (2001) 4316-4318.

[15]J. Zou, D. Kotchetkov, A.A. Balandin, D.I. Florescu, F.H. Pollak, J. Appl. Phys. 92 (2002) 2534-2539.

[16]S.I. Kim, K.H. Lee, H.A. Mun, H.S. Kim, S.W. Hwang, J.W. Roh, D.J. Yang, W.H. Shin, X.S. Li, Y.H. Lee, Science 348 (2015) 109-114.

[17]H.-S. Kim, S.D. Kang, Y. Tang, R. Hanus, G.J. Snyder, Mater. Horiz. 3 (2016) 234-240.

[18]M. Li, Z. Ding, Q. Meng, J. Zhou, Y. Zhu, H. Liu, M.S. Dresselhaus, G. Chen, arXiv:1605.05017 (2016).

[19]D.M. Rowe, V.S. Shukla, N. Savvides, Nature 290 (1981) 765-766.

[20]B. Poudel, Q. Hao, Y. Ma, Y. Lan, A. Minnich, B. Yu, X. Yan, D. Wang, A. Muto, D. Vashaee, Science 320 (2008) 634-638.

[21]G. Joshi, H. Lee, Y. Lan, X. Wang, G. Zhu, D. Wang, R.W. Gould, D.C. Cuff, M.Y. Tang, M.S. Dresselhaus, G. Chen, Z. Ren, Nano Lett. 8 (2008) 4670-4674.

[22]W. Xie, J. He, H.J. Kang, X. Tang, S. Zhu, M. Laver, S. Wang, J.R.D. Copley, C.M. Brown, Q. Zhang, T.M. Tritt, Nano Lett. 10 (2010) 3283-3289.

[23]G. Joshi, X. Yan, H. Wang, W. Liu, G. Chen, Z. Ren, Adv. Energy Mater. 1 (2011) 643-647.

[24]L.D. Chen, X.Y. Huang, M. Zhou, X. Shi, W.B. Zhang, J. Appl. Phys. 99 (2006) 064305.

[25]Q. Zhang, J. He, T.J. Zhu, S.N. Zhang, X.B. Zhao, T.M. Tritt, Appl. Phys. Lett. 93 (2008) 102109.

[26]M. Zhou, J.-F. Li, T. Kita, J. Am. Chem. Soc. 130 (2008) 4527-4532. 
[27]H. Li, X. Tang, Q. Zhang, C. Uher. Appl. Phys. Lett. 94 (2009) 102114.

[28]J. He, J.R. Sootsman, S.N. Girard, J.-C. Zheng, J. Wen, Y. Zhu, M.G. Kanatzidis, V.P. Dravid, J. Am. Chem. Soc. 132 (2010) 8669-8675.

[29]J. He, S.N. Girard, M.G. Kanatzidis, V.P. Dravid. Adv. Funct. Mater. 20 (2010) 764-772.

[30]L.-D. Zhao, S.-H. Lo, J. He, H. Li, K. Biswas, J. Androulakis, C.-I. Wu, T.P. Hogan, D.-Y. Chung, V.P. Dravid, J. Am. Chem. Soc. 133 (2011) 20476-20487.

[31]L.-D. Zhao, J. He, S. Hao, C.-I. Wu, T.P. Hogan, C. Wolverton, V.P. Dravid, M.G. Kanatzidis, J. Am. Chem. Soc. 134 (2012) 16327-16336.

[32]L.-D. Zhao, X. Zhang, H. Wu, G. Tan, Y. Pei, Y. Xiao, C. Chang, D. Wu, H. Chi, L. Zheng, S. Gong, C. Uher, J. He, M.G. Kanatzidis. J. Am. Chem. Soc. 138 (2016) 2366-2373.

[33]X. Liao, F. Zhou, E. Lavernia, D. He, Y. Zhu, Appl. Phys. Lett. 83 (2003) 5062-5064.

[34]M.W. Chen, E. Ma, K.J. Hemker, H.W. Sheng, Y.M. Wang, X.M. Cheng, Science 300 (2003) 1275-1277.

[35]L. Lu, Y. Shen, X. Chen, L. Qian, K. Lu, Science 304 (2004) 422-426.

[36]X. Zhang, A. Misra, H. Wang, M. Nastasi, J. Embury, T. Mitchell, R. Hoagland, J. Hirth, Appl. Phys. Lett. 84 (2004) 1096-1098.

[37]L. Lu, X. Chen, X. Huang, K. Lu. Revealing the Maximum Strength in Nanotwinned Copper, Science 323 (2009) 607-610.

[38]Y. Tian, B. Xu, D. Yu, Y. Ma, Y. Wang, Y. Jiang, W. Hu, C. Tang, Y. Gao, K. Luo, Z. Zhao, L.-M. Wang, B. Wen, J. He, Z. Liu, Nature 493 (2013) 385-388.

[39]A. Bagri, S.-P. Kim, R.S. Ruoff, V.B. Shenoy, Nano Lett. 11 (2011) 3917-3921.

[40]H. Zhan, Y. Zhang, J.M. Bell, Y. Gu, J. Phys. D Appl. Phys. 47 (2013) 015303.

[41]S. Li, X. Ding, J. Ren, X. Moya, J. Li, J. Sun, E.K.H. Salje, Sci. Rep. 4 (2014) 6375.

[42]S. Xiong, Y.A. Kosevich, K. Sääskilahti, Y. Ni, S. Volz, Phys. Rev. B 90 (2014) 195439.

[43]A. Porter, C. Tran, F. Sansoz, Phys. Rev. B 93 (2016) 195431.

[44]H. Dong, J. Xiao, R. Melnik, B. Wen, Sci. Rep. 6 (2016) 19575.

[45]T. Matsuo, H. Suzuki, J. Phys. Soc. Jpn. 43 (1977) 1974-1981.

[46]L.S. Mitchell, A. Anderson, J. Low Temp. Phys. 91 (1993) 341-369.

[47]D. Majumdar, S. Biswas, T. Ghoshal, J.D. Holmes, A. Singha, ACS Appl. Mater. Interfaces 7 (2015) 24679-24685.

[48]H. Fecht, E. Hellstern, Z. Fu, W. Johnson, Metal. Trans. A 21 (1990) 2333-2337.

[49]C. Koch, Y. Cho., Nanostructured Mater. 1 (1992) 207-212.

[50]C.C. Koch, Nanostructured Mater. 9 (1997) 13-22.

[51]J. Huang, Y. Wu, H. Ye, Acta Mater. 44 (1996) 1211-1221.

[52]J. Mao, Y. Wang, H.S. Kim, Z. Liu, U. Saparamadu, F. Tian, K. Dahal, J. Sun, S.

Chen, W. Liu, Z. Ren, Nano Energy 17 (2015) 279-289.

[53]Z. Liu, Y. Wang, W. Gao, J. Mao, H. Geng, J. Shuai, W. Cai, J. Sui, Z. Ren, Nano 
Energy 31 (2017) 194-200.

[54]H. Ehrenreich, J. Phys. Chem. Solids 2 (1957) 131-149.

[55]Y. Pei, Z.M. Gibbs, A. Gloskovskii, B. Balke, W.G. Zeier, G.J. Snyder., Adv. Energy Mater. 4 (2014).

[56]D.M. Rowe, C.M. Bhandari, Appl. Energy 6 (1980) 347-351.

[57]M.N. Luckyanova, J. Garg, K. Esfarjani, A. Jandl, M.T. Bulsara, A.J. Schmidt, A.J. Minnich, S. Chen, M.S. Dresselhaus, Z. Ren, Science 338 (2012) 936-939.

[58] M.N. Luckyanova, J. Mendoza, H. Lu, S. Huang, J. Zhou, M. Li, B.J. Kirby, A.J. Grutter, A.A. Puretzky, M.S. Dresselhaus, arXiv:1602.05057 (2016). 


\section{FIGURE CAPTIONS}

Figure 1. Comparison of microstructures between InSb specimens prepared by different methods. (a) SEM image shows the specimen with microscale grains, TEM images show the specimen (b) with nanoscale grains, (c) nanoscale grains with nanotwins, and (d)-(f) show the schematic views of microstructures of the specimens.

Figure 2. HRTEM and HAADF-STEM images of nanoscale twins. (a) Several nanoscale twins inside one of the grains, (b) magnified view of the rectangular region in (a), the inset shows one of the TBs, (c) several nanoscale twins with spacing of $\sim 3$ $\mathrm{nm}$, (d) multiple nanoscale twins with spacing of $\sim 1.5 \mathrm{~nm}$, (e) high magnification HAADF-STEM image shows one of the coherent TBs, the inset is the fast Fourier transform, and (f) magnified view of the rectangular region in (e).

Figure 3. Comparison of lattice thermal conductivity $\kappa_{\text {lat }}$ between different $\mathrm{InSb}$ specimens.

Figure 4. (a) Low magnification HADDF-STEM image showing a GB and several TBs, (b) magnified view of rectangular region 1 in (a), (c) filtered image of (b), and the extra half planes are pointed out by yellow arrows, (d) magnified view of rectangular region 3 in (b) shows the dislocations with closed right-hand Burgers circuits marked, (e) magnified view of rectangular region 4 in (b) shows the intrinsic 
stacking faults. The capitals A, B and C and lower-case a, b, c denote the stacking sequence of $\mathrm{Sb}$ and In atomic layers, (f) magnified view of rectangular region 2 in (a) shows the GB region marked by dash lines. 


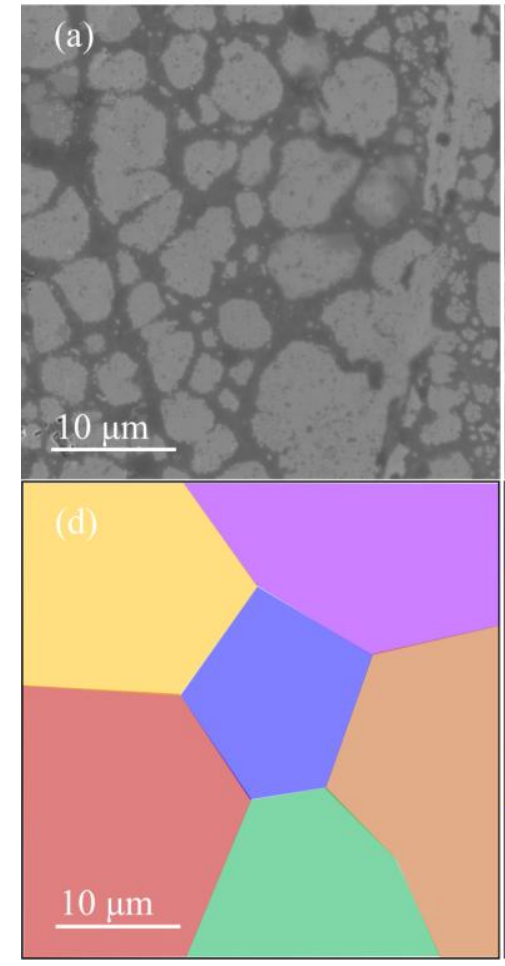

Microscale grains

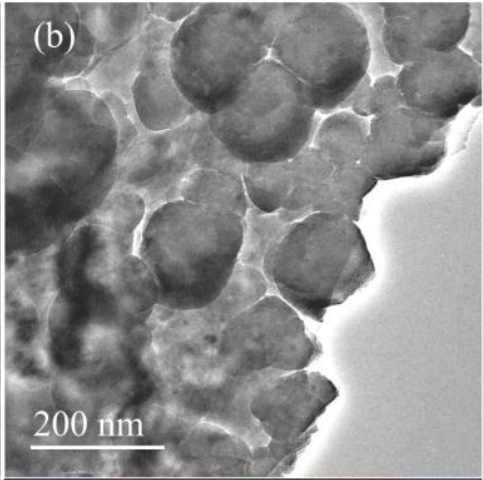

(e)

$200 \mathrm{~nm}$

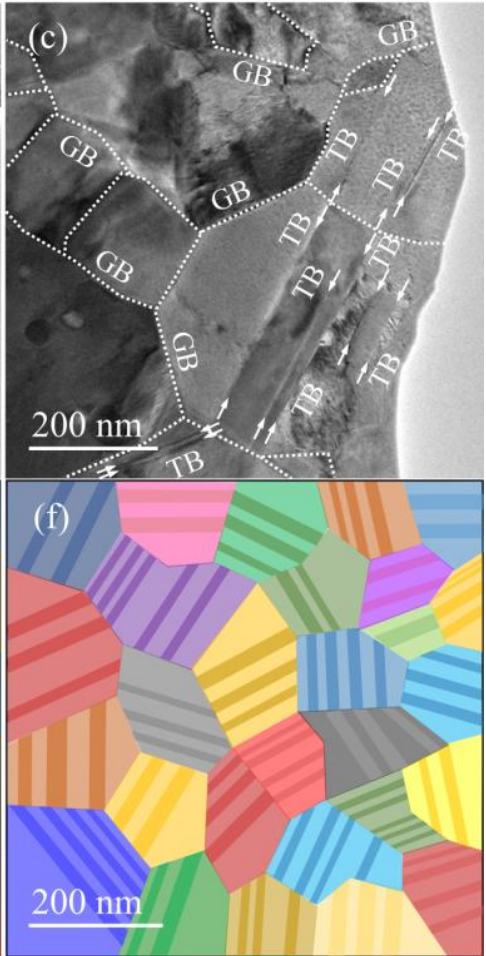

Nanoscale grains + Nanotwins

Nanoscale grains 


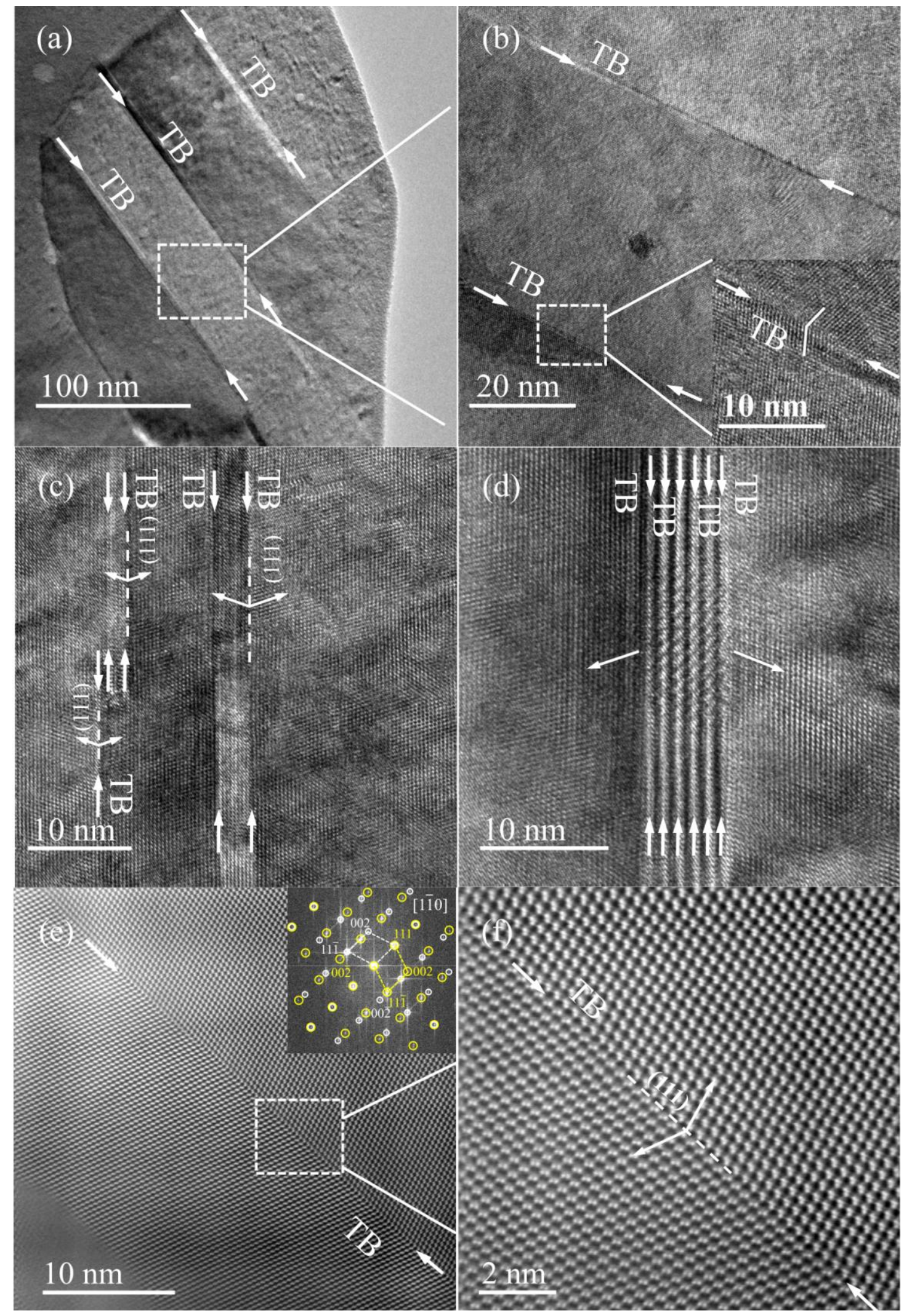




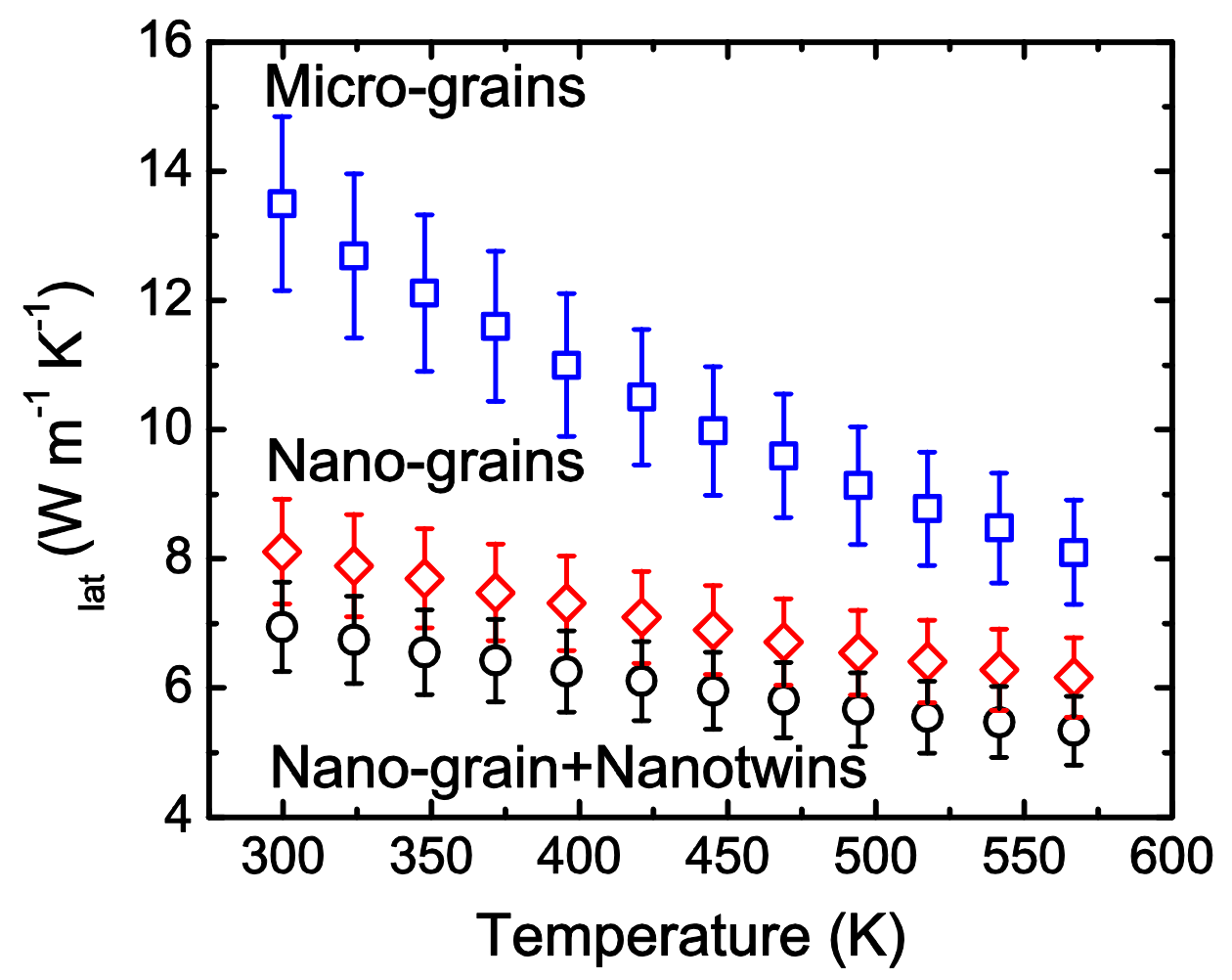




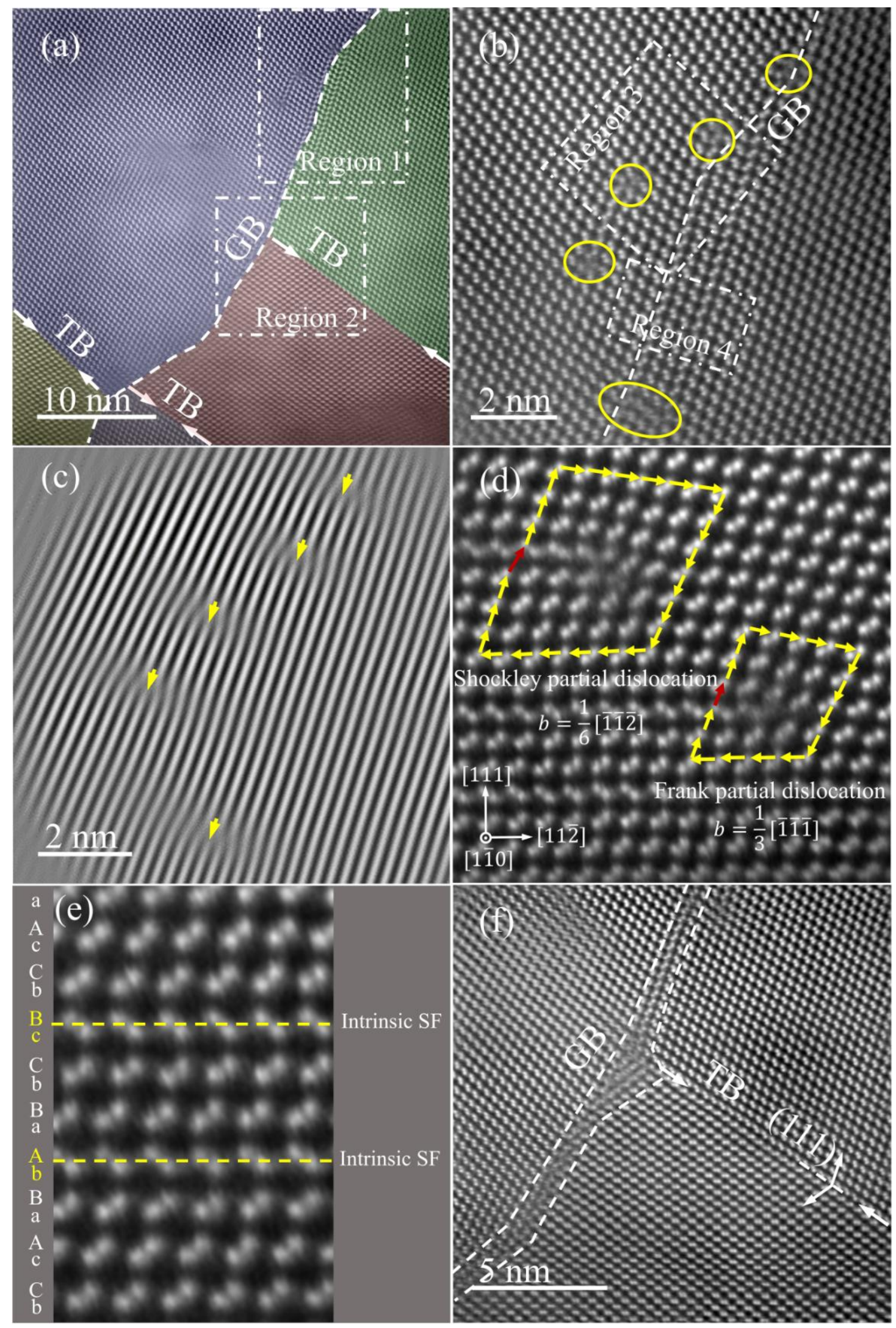




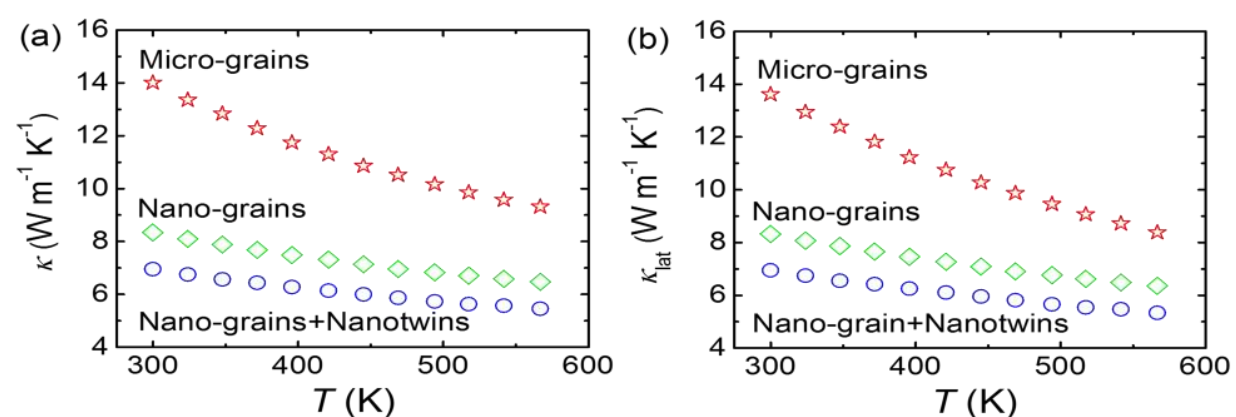

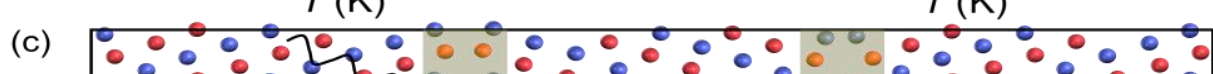

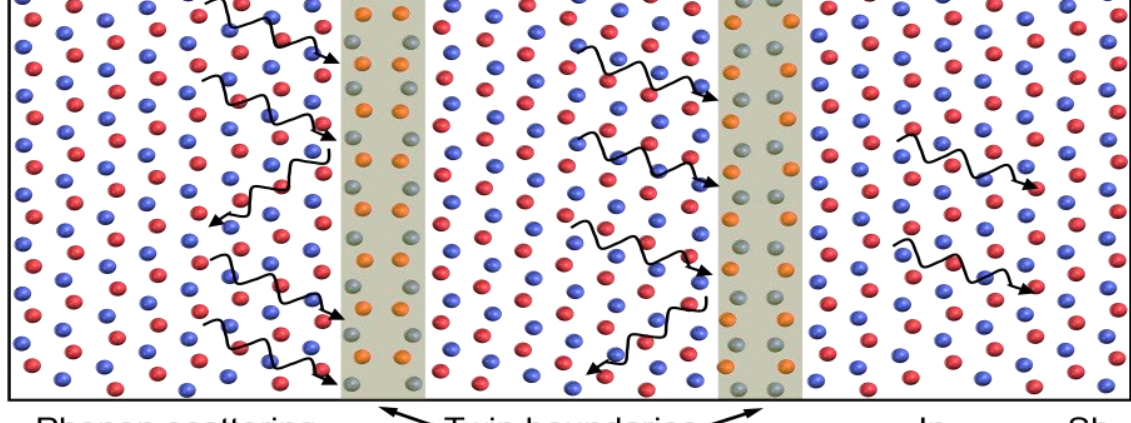

Phonon scattering Twin boundaries $\longrightarrow \mathrm{In} \quad \mathrm{Sb}$ 